\title{
A NOTE ON SIMPLE ANTI-COMMUTATIVE ALGEBRAS OBTAINED FROM REDUCTIVE HOMOGENEOUS SPACES
}

\author{
ARTHUR A. SAGLE
}

1. Basics. Let $G$ be a connected Lie group and $H$ a closed subgroup, then the homogeneous space $M=G / H$ is called reductive if there exists a decomposition $\mathfrak{g}=\mathfrak{m}+\mathfrak{h}$ (subspace direct sum) with $[\mathfrak{m}, \mathfrak{h}] \subset \mathfrak{m}$ where $\mathfrak{g}$ (resp. $\mathfrak{b}$ ) is the Lie algebra of $G$ (resp. $H$ ); in this case the pair $(\mathfrak{g}, \mathfrak{h})$ is called a reductive pair. For $x, y \in \mathfrak{m}$ an anti-commutative multiplication $x y$ is defined in $\mathfrak{m}$ by $[x y]=x y+h(x, y)$ where $x y$ (resp. $h(x, y))$ is the component of $[x y] \in \mathfrak{g}$ in $\mathfrak{m}$ (resp. $\mathfrak{h}$ ) relative to a fixed decomposition $\mathfrak{g}=\mathfrak{m} \mathfrak{\mathfrak { h }}$. The study of these algebras is motivated by the work of K. Nomizu where this multiplication is related to the canonical $G$-invariant connection $\nabla$ of the first kind on $G / H$ by $\left[\nabla_{x^{*}}\left(y^{*}\right)\right] p_{0}=\frac{1}{2} x y$ where $p_{0}=H \in M$. There is a correspondence between holonomy irreducible non-symmetric spaces $M$ and simple algebras $\mathfrak{m}$ with the above connection and multiplication, see. [11]. Using this the holonomy algebra is easily determined by showing certain derivations induced by $h(x, y) \in \mathfrak{h}$ are inner. Thus in this paper we study the simplicity of the algebra $\mathrm{m}$ and its derivations subject to "irreducibility" conditions on $\mathfrak{g}, \mathfrak{h}$ and $\mathfrak{n}$; in particular when $\mathfrak{m}$ is $\mathfrak{h}$-irreducible and when $\mathfrak{g}$ and $\mathfrak{h}$ are simple Lie algebras. Applications are given to Lie admissible algebras and to homogeneous spaces $M$ with a group-like (non-associative) mulțiplication.

The Lie algebra identities in $g$ yield the following identities for $\mathfrak{m}$ and $\mathfrak{h}$.

(2) $\quad h(x, y)=-h(y, x)$

(3) $[x h(y, z)]+[y h(z, x)]+[z h(x, y)]=J(x, y, z)$

(4) $\quad h(x y, z)+h(y z, x)+h(z x, y)=0$

Received July 21, 1966.

Sponsored by NSF Grant GP 1453 and by the Office of Naval Research. 


$$
[h(x, y) h]=h([x h], y)+h\left(x,\left[\begin{array}{ll}
y & h
\end{array}\right]\right)
$$$$
\left[\begin{array}{ll}
x y & h
\end{array}\right]=\left[\begin{array}{ll}
x & h
\end{array}\right] y+x\left[\begin{array}{ll}
y & h
\end{array}\right]
$$

where $J(x, y, z)=(x y) z+(y z) x+(z x) y$. Thus if $\mathfrak{h}(\mathfrak{m}, \mathfrak{m})=$ linear space spanned by all the $h(x, y)$ 's, we have from (5) that $\mathfrak{h}(\mathfrak{m}, \mathfrak{m})$ is an ideal in $\mathfrak{h}$ and from (6) that the mappings $D(h)=a d_{\mathrm{m}} h$ are contained in the derivation algebra $D(\mathfrak{m})$ of $\mathfrak{m}$. From these we see that $\mathfrak{m}+\mathfrak{h}(\mathfrak{m}, \mathfrak{m})$ is an ideal in $\mathfrak{g}$ and if there exists an ideal $\mathfrak{k}$ of $g$ such that $\mathfrak{g}=\mathfrak{m}+\mathfrak{h}(\mathfrak{m}, \mathfrak{m})+\mathfrak{t}$ (e.g. $\mathfrak{g}$ semi-simple) then the derivations induced by $\mathfrak{f}$ are zero $([\mathfrak{m} \mathfrak{f}] \subset \mathfrak{m} \cap \mathfrak{l}=0$ using $\mathfrak{t}$ is an ideal of $\mathfrak{g}$ and $(\mathfrak{g}, \mathfrak{h})$ is a reductive pair). These considerations lead to the study of the "minimal" reductive pair $(\mathfrak{m}, \mathfrak{h}(\mathfrak{m}, \mathfrak{m})$ ) and if we write $D(x, y)=a d_{\mathfrak{m}} h(x, y)$ then the relations (1)-(6) can be rewritten in terms of these derivations of $\mathfrak{m}$ to yield the axioms of a general Lie triple system (g.L.t.s.) for $\mathfrak{g}=\mathfrak{m}+\mathfrak{h}[9,11,13]$

$$
\begin{aligned}
& x y=-y x \\
& D(x, y)=-D(y, x) \\
& x D(y, z)+y D(z, x)+z D(x, y)=J(x, y, z) \\
& D(x y, z)+D(y z, x)+D(z x, y)=0 \\
& {[D(w, x), D(y, z)]=D(w D(y, z), x)+D(w, x D(y, z))} \\
& (x y) D(u, v)=(x D(u, v)) y+x(y D(u, v))
\end{aligned}
$$

where $[A, B]=A B-B A$.

Note that when $x y \equiv 0$ for all $x, y \in \mathfrak{m}$ then $\mathfrak{m}+\mathfrak{h}(\mathfrak{m}, \mathfrak{m})$ is a L.t.s. where $[x, y, z]=x D(y, z)$ is the trilinear operation on $\mathfrak{m}[6]$.

There are many examples of g.L.t.s. (section 2) but we shall be primarily concerned with a g.L.t.s. $\mathfrak{m}+\mathfrak{h}(\mathfrak{m}, \mathfrak{m})$ where $\mathfrak{m}$ is $\mathfrak{h}(\mathfrak{m}, \mathfrak{m})$-irreducible or $\mathfrak{m}$ is a simple algebra. Algebraically this is motivated by the seven dimensional Malcev algebra in [8] which gives rise to the g.L.t.s. $\mathfrak{g}=\mathfrak{m}+\mathfrak{h}(\mathfrak{m}, \mathfrak{m})$ where $\mathrm{g}$ is of type $B_{3}$, $\mathfrak{h}$ of type $G_{2}$ [14] $\mathfrak{m}$ is $\mathfrak{h}$-irreducible and a simple algebra. We shall assume throughout this paper that the base field $F$ is of characteristic zero and in section 2 we shall show that if $(\mathfrak{g}, \mathfrak{h})$ is a reductive pair with $\mathfrak{g}=\mathfrak{m}+\mathfrak{h}$ where $\mathfrak{m}$ is $\mathfrak{h}$-irreducible and $\mathfrak{m} \mathfrak{m} \neq 0$, then $\mathfrak{m}$ is a simple algebra and $a d_{\mathfrak{m}}$ is semi-simple under certain conditions (if $F$ is algebraically closed). We also consider the reductive pair $(\mathfrak{g}, \mathfrak{h})$ where $\mathfrak{g}$ and $\mathfrak{h}$ are simple Lie algebras; in this case if $\mathfrak{m m} \neq 0$, then $\mathfrak{m}$ is simple and the derivations induced by $\mathfrak{h}$ are inner. 
In [6] the analysis of a L.t.s. $\mathfrak{T}$ depends on the study of automorphisms of the Lie algebra $\mathfrak{L}=\mathfrak{T} \oplus[\mathfrak{T} \mathfrak{T}]$ which are of period 2 . We now describe this for the more general case of a reductive pair $(\mathfrak{g}, \mathfrak{h})$.

Proposition 1. Let $\mathrm{g}$ be a Lie algebra and $\mathfrak{h}$ a subspace of $\mathrm{g}$. Let $s$ be an endomorphism of $\mathrm{g}$ such that $s^{2}=I$ and $s$ is a maximal $\mathfrak{h}$-endomorphism i.e. $h s=h$ for all $h \in \mathfrak{h},[l h] s=[l s h]$ for all $l \in \mathfrak{g}, h \in \mathfrak{h}$ and $\mathfrak{h}=\{l \in \mathfrak{g}: l s=l\}$. Then if $\mathfrak{m}=\{l \in \mathfrak{g}: l s=-l\}$ we have $(\mathfrak{g}, \mathfrak{h})$ is a reductive pair which uniquely determines $s$. Furthermore if $p=x+h, q=y+k, \mathfrak{g}=\mathfrak{m}+\mathfrak{h}$, then [ps qs]$\left[\begin{array}{ll}p & q\end{array}\right]=2 x y$; thus $\mathrm{g}$ is a L.t.s. if and only if $s$ is an automorphism of $\mathrm{g}$ if and only if $\mathrm{m}^{2} \equiv 0$.

Proof. Since $s^{2}=I$ we have $\mathfrak{g}=\mathfrak{v}_{1}+\mathfrak{v}_{2}$ where $\mathfrak{v}_{j}=\left\{l \in \mathfrak{g}: l s=(-1)^{j+1} l\right\}$ so that $\mathfrak{h}=\mathfrak{v}_{1}, \mathfrak{m}=\mathfrak{y}_{2}$ and $\mathfrak{g}=\mathfrak{m}+\mathfrak{h}$. Next $[\mathfrak{m} \mathfrak{h}] \subset \mathfrak{m}$ and $[\mathfrak{h} \mathfrak{h}] \subset \mathfrak{h}$; for if $x \in \mathfrak{m}, h \in \mathfrak{h}$, then $\left[\begin{array}{ll}x & h\end{array}\right] s=\left[\begin{array}{ll}x s & h\end{array}\right]=-\left[\begin{array}{ll}x & h\end{array}\right]$ and similarly $[\mathfrak{h} \mathfrak{h}] \subset \mathfrak{h}$. Conversely if $(\mathfrak{g}, \mathfrak{h})$ is a reductive pair and $\mathfrak{g}=\mathfrak{m}+\mathfrak{h}$, then the endomorphism $s: x+h$ $\rightarrow-x+h$ is a maximal $\mathfrak{h}$-endomorphism so that $s^{2}=I$. Furthermore if $\mathfrak{g}=\mathfrak{m}+\mathfrak{h}$ is determined by two $\mathfrak{h}$-endomorphism $s$ and $t$ as above, then clearly $s=t$. The formula $\left[\begin{array}{ll}p s & q s\end{array}\right]-\left[\begin{array}{ll}p & q\end{array}\right] s=2 x y$ is a straightforward computation.

We shall want to consider Lie algebras $g$ which are semi-simple and as an application of the above result we have the following theorem.

Theorem 2. Let $\mathfrak{g}=\mathfrak{m}+\mathfrak{h}$ be a semi-simple g.L.t.s. (i.e. $\mathfrak{h}=\mathfrak{h}(\mathfrak{m}, \mathfrak{m}))$ defined by the involution $s$ previously discussed, let $\mathfrak{m}$ be $\mathfrak{h}$-irreducible and assume $a d_{\mathrm{m}} h=0$ implies $h=0$, then

$\mathrm{g}$ is simple or

(2) $\mathfrak{g}=g_{1}+g_{2}$ where $g_{1}$ is as imple ideal of $\mathfrak{g}$ and $g_{2}=g_{1} s$. If $g$ is not simple, we may write $\mathfrak{g}=\mathfrak{g}_{1} \oplus \mathfrak{g}_{2}$ where $\mathfrak{g}_{1}$ and $\mathfrak{g}_{2}$ are simple ideals and dim. $\mathfrak{g}_{1}=$ dim. $\mathfrak{g}_{2}$. In case $\mathfrak{h}$ is simple dim. $\mathfrak{h}=$ dim. $\mathfrak{m}=$ dim. $\mathfrak{g}_{1}$.

Proof. Let $\mathfrak{g}=\mathfrak{m}+\mathfrak{h}=\oplus \Sigma \mathfrak{g}_{k}$ be semi-simple defined by the involution $s$ i.e. $s^{2}=I,\left[a d_{g} h, s\right]=0$, then $\mathfrak{t}_{j}=\mathfrak{g}_{j}+\mathfrak{g}_{j} s$ is $a d_{g} \mathfrak{h}$-invariant subspace of $\mathfrak{g}:\left[\mathfrak{g}_{j} \mathfrak{h}\right] \subset \mathfrak{g}_{j}$ since $\mathfrak{g}_{j}$ is an ideal of $\mathfrak{g}$ and $\left[\mathfrak{g}_{j} s \mathfrak{h}\right]=\mathfrak{g}_{j} s$ ad $\mathfrak{h}=\mathfrak{g}_{j}$ ad $\mathfrak{h} s=\left[\mathfrak{g}_{j} \mathfrak{h}\right] s \subset \mathfrak{g}_{j} s$.

Next we have $\mathfrak{g}_{j}$ is not an ideal in $\mathfrak{h}$. For suppose $\mathfrak{g}_{j}$ is an ideal in $\mathfrak{h}$, then $\left[\mathfrak{m} \mathfrak{g}_{j}\right] \subset[\mathfrak{m} \mathfrak{h}] \subset \mathfrak{m}$, using $(\mathfrak{g}, \mathfrak{h})$ is reductive. But since $\mathfrak{g}_{j}$ is an ideal of $\mathfrak{g},\left[\mathfrak{m} \mathfrak{g}_{j}\right] \subset \mathfrak{g}_{j} \subset \mathfrak{h}$ and therefore $\left[\mathfrak{m} \mathfrak{g}_{j}\right] \subset \mathfrak{m} \cap \mathfrak{h}=0$. Thus $a d_{\mathfrak{m}} \mathfrak{g}_{j}=0$ 
which implies $\mathfrak{g}_{i}=0$. Now since $\mathfrak{g}_{j}$ is not an ideal of $\mathfrak{h}$, there exists $x+h \in \mathfrak{g}_{j}$ with $0 \neq x \in \mathfrak{m}, h \in \mathfrak{h}$. But then $-x+h=(x+h) s \in \mathfrak{g}_{j} s$ and therefore

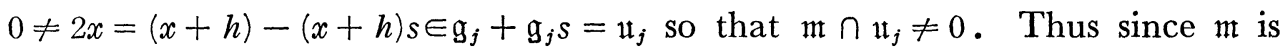
h-irreducible, $\mathfrak{m}=\mathfrak{m} \cap \mathfrak{t}_{j} \subset \mathfrak{t}_{j}$.

Now we shall show $\mathfrak{h}=\mathfrak{h}(\mathfrak{m}, \mathfrak{m}) \subset \mathfrak{u}_{j}$ and therefore $\mathfrak{g}=\mathfrak{m} \dot{+} \mathfrak{h}=\mathfrak{u}_{j}$. For any $x, y \in \mathfrak{m}$, there exists $z \in \mathfrak{m}$ so that $z+h(x, y) \in \mathfrak{g}_{j}$ where $[x y]=x y+h(x, y)$. For let $\mathfrak{m}_{j}=\left\{x \in \mathfrak{m}\right.$ : there exists $h \in h$ with $\left.x+h \in \mathfrak{g}_{j}\right\}=$ projection of $\mathfrak{g}_{j}$ into $\mathfrak{m}$. Then $\mathfrak{m t}_{j} \neq 0$, otherwise $\mathfrak{g}_{j}$ is an ideal contained in $\mathfrak{h}$; also $\mathfrak{m}_{j}$ is $\mathfrak{h}$ invariant subspace of $\mathfrak{m}$ and so equals $\mathfrak{m}$. Now to find the desired $z$ we have for $x, y \in \mathfrak{m}, h \in \mathfrak{h}$ with $x+h \in \mathfrak{g}_{j}$ that $[y x+h] \in \mathfrak{g}_{j}$ and from this we have for $z=y x+\left[\begin{array}{ll}y & h\end{array}\right] \in \mathfrak{m}$ that $z+h(y, x) \in \mathfrak{g}_{j}$. Next using this and $\mathfrak{m} \subset \mathfrak{u}_{j}=\mathfrak{g}_{j}+\mathfrak{g}_{j} s$ we have for any $h(x, y) \in \mathfrak{h}=\mathfrak{h}(\mathfrak{m}, \mathfrak{m})$, that there exists $z \in \mathfrak{m}$ with $z+h(x, y) \in g_{j}$ and therefore $h(x, y) \in \mathfrak{m}+\mathfrak{g}_{j} \subset \mathfrak{u}_{j} . \quad$ Thus $\mathfrak{h} \subset \mathfrak{u}_{j}$ so that $\mathfrak{g}=\mathfrak{g}_{j}+\mathfrak{g}_{j} s$.

Two cases arise (1) $\mathfrak{g}=\mathfrak{g}_{j}$ i.e. $j=1$ so that $\mathfrak{g}=\mathfrak{n} \mathfrak{f} \mathfrak{h}$ is actually simple or (2) $g$ properly contains $g_{j}$. In this case we shall show $\mathfrak{g}=\mathfrak{g}_{j}+\mathfrak{g}_{j} s$. Let $x+h \in \mathfrak{g}_{j} \cap \mathfrak{g}_{j} s$ where $x \in \mathfrak{m}, h \in \mathfrak{h}$, then $-x+h=(x+h)_{s} \in\left(\mathfrak{g}_{j} s\right) s=\mathfrak{g}_{j}$; but since $x+h \in g_{j}$ also, $2 x=(x+h)-(-x+h) \in g_{j}$. Thus if $x \neq 0, \mathfrak{m} \cap g_{j}$ is a nonzero $\mathfrak{h}$-invariant subspace of $\mathfrak{m}$ which must equal $\mathfrak{m}$. Thus $\mathfrak{m}=\mathfrak{m} \cap \mathfrak{g}_{j} \subset \mathfrak{g}_{j}$ and by the argument in the preceding paragraph $\mathfrak{h}=\mathfrak{h}(\mathfrak{m}, \mathfrak{m}) \subset \mathfrak{g}_{j}$. Therefore $\mathfrak{g}=\mathfrak{g}_{j}$, contrary to the choice of $\mathrm{g}$ in case 2 ; thus $x=0$ and $h \in \mathfrak{g}_{j} \cap \mathfrak{g}_{j} s$. Next for any $y \in \mathfrak{m}, y_{\mathfrak{m}} h=\left[\begin{array}{ll}y & h\end{array}\right] \in \mathfrak{m}$; but since $h \in \mathrm{g}_{j}$ which is an ideal of $\mathfrak{g},\left[\begin{array}{ll}y & h\end{array}\right] \in \mathfrak{g}_{j}$. Thus $\left[\begin{array}{ll}y & h\end{array}\right] \in \mathfrak{g}_{j} \cap \mathfrak{m}$ so that if $[y h] \neq 0, \mathfrak{g}_{j} \cap \mathfrak{m}$ is a nonzero $\mathfrak{h}$-invariant subspace which must equal $\mathfrak{m}$. Thus again $\mathfrak{m} \subset \mathfrak{g}_{j}$ and we may conclude $\mathrm{g}=\mathrm{g}_{j}$, contrary to case 2 . Therefore $a d_{\mathfrak{m}} h=0$ which implies $h=0$ so that $\mathfrak{g}=\mathfrak{g}_{j}+\mathfrak{g}_{j} s$.

Next let $\mathfrak{g}_{1}$ be a simple ideal of minimal dimension and write $\mathfrak{g}=\mathfrak{g}_{1} \oplus \mathfrak{g}_{2}$ where $g_{2}$ is a semi-simple ideal of $g$. If $g_{2}$ is not simple, then there is a simple ideal $\mathfrak{p}$ of $\mathfrak{g}$ in $\mathfrak{g}_{2}$ and dim. $\mathfrak{p}<\operatorname{dim} . \mathfrak{g}_{2}=\operatorname{dim} . \mathfrak{g}-\operatorname{dim} . \mathfrak{g}_{1}=\operatorname{dim}$. $\left(g_{1}+g_{1} s\right)$ - dim. $g_{1}=$ dim. $g_{1}$; a contradiction to the choice of $g_{1}$.

Now if $\mathfrak{h}$ is simple, we see from the definition of $\mathfrak{h}$ in terms of the map $s$ that $\left\{u+u s: u \in \mathfrak{g}_{1}\right\}$ is a non-zero $\mathfrak{h}$-invariant subspace of $\mathfrak{h}$; thus it must equal $\mathfrak{h}$. Similarly $\left\{u-u s: u \in g_{1}\right\}=\mathfrak{m}$. If $u_{1}, \ldots, u_{t}$ is a basis for $g_{1}$, then $\left\{u_{i}+u_{i} s: i=1, \ldots, t\right\}$ clearly spans $\mathfrak{h}$. If $\sum a_{i}\left(u_{i}+u_{i} s\right)=0$, then for $v=\sum a_{i} u_{i}$ we have $v s=-v$ so that $v \in \mathfrak{g}_{1} \cap \mathfrak{m}$. If $v \neq 0$, then $\mathfrak{g}_{1} \cap \mathfrak{m}$ is a non-zero $\mathfrak{h}$-invariant subspace of $\mathfrak{m}$ and therefore $\mathfrak{m}=\mathfrak{g}_{1} \cap \mathfrak{m}$. But then for $x, y \in \mathfrak{m} \subset g_{1}$, we have $h(x, y)=[x y]-x y \in g_{1}$ so that $g \subset g_{1}$; contrary to the 
choice of $g$ not simple. Thus $v=0$ so that $a_{i}=0$ and $\left\{u_{i}+u_{i} s\right\}$ are independent. From this $\operatorname{dim} \cdot \mathfrak{h}=\operatorname{dim} . \mathfrak{g}_{1}$. Similarly for $\mathfrak{m}$ we have $\left\{u_{i}-u_{i} s: i=1, \ldots, t\right\}$ spans $\mathfrak{m}$ and if $\sum b_{i}\left(u_{i}-u_{i} s\right)=0$, then for $w=\sum b_{i} u_{i}$ we have $w s=w$. Thus $w \in \mathfrak{h} \cap \mathfrak{g}_{1}$ and if $w \neq 0$ we have by the simplicity of $\mathfrak{h}$ that $\mathfrak{h}=\mathfrak{h} \cap \mathfrak{g}_{1} \subset \mathfrak{g}_{1}$. By hypothesis [ $\mathfrak{m} \mathfrak{h}$ ] is a non-zero ( $\mathfrak{h}$-invariant) subspace of $\mathfrak{m}$ so we have $\mathfrak{m}=[\mathfrak{m} \mathfrak{h}] \subset\left[\mathfrak{m} \mathfrak{g}_{1}\right] \subset \mathfrak{g}_{1}$. Thus as before we obtain the contradiction that $\mathfrak{g}=\mathfrak{m}+\mathfrak{h} \subset \mathfrak{g}_{1}$, which is simple.

Thus the practical attempt to find algebras $\mathfrak{m}$ which are $\mathfrak{h}$-irreducible reduces to considering some well behaved Lie algebras $\mathfrak{g}$. If $\mathfrak{h}$ is simple we have the following criterion which reduces the attempt to considering simple Lie algebras $g$.

TheOREM 3. Let $\mathfrak{g}=\mathfrak{m}+\mathfrak{h}$ be g.L.t.s. over an algebraically closed field where $\mathfrak{m}$ is $\mathfrak{h}$-irreducible, $\mathfrak{h}=\mathfrak{h}(\mathfrak{m}, \mathfrak{m})$ is simple and $[\mathfrak{m} \mathfrak{h}] \neq 0$. Let $\mathfrak{h}_{0}$ be a Cartan subalgebra of $\mathfrak{h}$ and let $\Sigma$ be the set of roots of $\mathfrak{h}_{0}$ in $\mathfrak{h}$ and $\Delta$ be the set of weights of $\mathfrak{h}_{0}$ in $\mathfrak{m}$. If $\Sigma \neq \Delta$, then $\mathfrak{g}$ is simple.

Proof. Let $\mathfrak{b}$ be a proper ideal of $\mathfrak{g}$ and let $\pi_{\mathfrak{m}}: \mathfrak{g} \rightarrow \mathfrak{m}, \pi_{\mathfrak{h}}: \mathfrak{g} \rightarrow \mathfrak{h}$ be the projections defined by the (fixed) decomposition $g=\mathfrak{m}+\mathfrak{h}$. Then

$$
\begin{aligned}
& \mathfrak{b} \pi_{\mathfrak{m}}=\{x \in \mathfrak{m}: \text { there exists } h \in \mathfrak{h} \text { and } x+h \in \mathfrak{b}\}=0 \text { or } \mathfrak{m}, \\
& \text { and } \mathfrak{b} \cap \mathfrak{m}=0 \text { or } \mathfrak{m} ; \\
& \mathfrak{b} \pi_{\mathfrak{h}}=\{h \in \mathfrak{h}: \text { there exists } x \in \mathfrak{m} \text { and } x+h \in \mathfrak{b}\}=0 \text { or } \mathfrak{h}, \\
& \text { and } \mathfrak{b} \cap \mathfrak{h}=0 \text { or } \mathfrak{h} .
\end{aligned}
$$

Both of these follow from the $\mathfrak{h}$-irreducibility of $\mathfrak{m}$ and $\mathfrak{h}$. For the first let $x \in \mathfrak{b} \pi_{\mathfrak{m}}$ and $k \in \mathfrak{h}$ so that $b=x+k \in \mathfrak{b}$. Then for any $h \in \mathfrak{h},\left[\begin{array}{ll}x & h\end{array}\right]+\left[\begin{array}{ll}k & h\end{array}\right]=\left[\begin{array}{ll}b & h\end{array}\right] \in \mathfrak{b}$. Thus $[x h] \in \mathfrak{b} \pi_{\mathfrak{m}}$ since $[k h] \in \mathfrak{h}$ is such that $[x h]+\left[\begin{array}{ll}k & h\end{array}\right] \in \mathfrak{b}$. Therefore $\mathfrak{b} \pi_{\mathfrak{m}}$ is $\mathfrak{h}$-invariant subspace of $\mathfrak{m}$ and so equals 0 or $\mathfrak{m}$. Similarly for the statement concerning $\mathfrak{h}$.

Now if $\mathfrak{b} \cap \mathfrak{m}=\mathfrak{m}$, then $\mathfrak{b} \pi_{\mathfrak{m}}=\mathfrak{m}$; for if $x \in \mathfrak{m}=\mathfrak{b} \cap \mathfrak{m} \subset \mathfrak{b}$, then, by definition, $x \in \mathfrak{b} \pi_{\mathfrak{m}}$. Therefore for any $x, y \in \mathfrak{m} \subset \mathfrak{b},[x \quad y]=x y+h(x, y) \in \mathfrak{b}$ and since $x y \in \mathfrak{b} \pi \mathfrak{m}=\mathfrak{m} \subset \mathfrak{b}$ we have $h(x, y)=[x y]-x y \in \mathfrak{b} . \quad$ Thus $\mathfrak{h}=\mathfrak{h}(\mathfrak{m}, \mathfrak{m}) \subset \mathfrak{b}$ so that $\mathfrak{g}=\mathfrak{m} \dot{+} \mathfrak{h} \subset \mathfrak{b}$, contrary to the choice of $\mathfrak{b}$ as a proper ideal. Therefore $\mathfrak{b} \cap \mathfrak{m}=0$. Similarly if $\mathfrak{b} \cap \mathfrak{h} \subset \mathfrak{h}$, then $\mathfrak{b} \pi_{\mathfrak{h}}=\mathfrak{h}$. But since $[\mathfrak{m} \mathfrak{h}] \neq 0$ and $[\mathfrak{m} \mathfrak{h}]$ is $\mathfrak{h}$-invariant it therefore equals $\mathfrak{m}$. Thus $\mathfrak{h}=\mathfrak{b} \cap \mathfrak{h} \subset \mathfrak{b}$ and $\mathfrak{m}=[\mathfrak{m} \mathfrak{h}] \subset[\mathfrak{m} \mathfrak{b}] \subset \mathfrak{b}$ so that $\mathfrak{g}=\mathfrak{b}$; therefore $\mathfrak{b} \cap \mathfrak{h}=0$. These two facts 
mean that if $0 \neq x+h \in \mathfrak{b}$, then $x \neq 0$ and $h \neq 0$ otherwise $\mathfrak{b} \cap \mathfrak{h} \neq 0$ or $\mathfrak{m} \cap \mathfrak{h} \neq 0$. Thus $\mathfrak{b} \pi_{\mathfrak{m}}=\mathfrak{m}$ and $\mathfrak{b} \pi_{\mathfrak{h}}=\mathfrak{h}$.

Next decompose $\mathfrak{m}$ and $\mathfrak{h}$ relative to $\mathfrak{h}_{0}$ into weight and root spaces: $\mathfrak{m}=\mathfrak{m}_{0}+\sum_{\Gamma \in \Delta} \mathfrak{m}_{\Gamma}$ and $\mathfrak{h}=\mathfrak{h}_{0}+\sum_{\rho \in \Sigma} \mathfrak{h}_{\rho}$. For $\rho \in \Sigma$ and $0 \neq h_{\rho} \in \mathfrak{h}=\mathfrak{b} \pi_{\mathfrak{h}}$ (where $\left.\mathfrak{h}_{\rho}=h F_{\rho}\right)$, there exists $x \in \mathfrak{m}$ with $x+h_{\rho} \in \mathfrak{b}$ and therefore for $h_{0} \in \mathfrak{h}_{0}$ we have $\left[x h_{0}\right]+\rho\left(h_{0}\right) h_{\rho}=\left[x+h_{\rho} h_{0}\right] \in \mathfrak{b}$ and also $\rho\left(h_{0}\right)\left(x+h_{\rho}\right) \in \mathfrak{b}$. Thus $\rho\left(h_{0}\right) x-\left[x h_{0}\right]=$ $\rho\left(h_{0}\right)\left(x+h_{\rho}\right)-\left[x+h_{\rho} h_{0}\right] \in \mathfrak{b} \cap \mathfrak{m}=0$ so that $\left[x h_{0}\right]=\rho\left(h_{0}\right) x$ and therefore $\rho \in \Delta$. Conversely let $\Gamma \in \Delta$ and $0 \neq x \in \mathfrak{m}_{\Gamma} \subset \mathfrak{m}=\mathrm{b} \pi \mathfrak{m}$, then there exists $0 \neq h \in \mathfrak{h}$ with $x+h \in \mathfrak{b}$ and therefore $\Gamma\left(h_{0}\right) x+\left[h h_{0}\right]=\left[x+h h_{0}\right] \in \mathfrak{b}$ so that $\left[h h_{0}\right]-\Gamma\left(h_{0}\right) h=$ $\left[x+h h_{0}\right]-\Gamma\left(h_{0}\right)(x+h) \in \mathfrak{b} \cap \mathfrak{h}=0$ and therefore $\Gamma \in \Sigma$. Thus $\Delta=\Sigma$ when $\mathfrak{b}$ is a proper ideal. This proof can be modified when $\mathfrak{h}$ is a split Lie algebra.

We shall show that this is the best possible result of this type by constructing a six dimensional Lie algebra $\mathfrak{g}=\mathfrak{m}+\mathfrak{h}$ where $\mathfrak{h}$ is simple, $\mathfrak{m}$ is $\mathfrak{h}$-irreducible, $\mathfrak{m}$ is simple, $\Sigma=\Delta$ yet $\mathfrak{g}$ is not semi-simple. First we consider reductive Lie algebras with radical.

Theorem 4. Let $\mathfrak{g}=\mathfrak{m}+\mathfrak{h}$ be g.L.t.s where $\mathfrak{h}=\mathfrak{h}(\mathfrak{m}, \mathfrak{m})$ is semi-simple, $\mathfrak{m}$ is $\mathfrak{h}$-irreducible and the radical $\mathfrak{f}$ of $\mathfrak{g}$ is nonzero. Then $\mathfrak{g}=\mathfrak{i}+\mathfrak{m}=\mathfrak{i}+\mathfrak{h}$ and [f $\mathfrak{f}]=0$. Thus in particular $\operatorname{dim} . \mathfrak{h}=\operatorname{dim} . \mathfrak{m}=\operatorname{dim} . \mathfrak{f}=1 / 2 \operatorname{dim} . \mathfrak{g}$.

Proof. Let $\mathfrak{r}$ be any solvable nonzero ideal of $g$, then $\mathfrak{r} \pi \mathfrak{m}$ and $x_{\mathfrak{h}}$ are $\mathfrak{h}$-invariant subspaces i.e. $\mathfrak{r}_{\mathfrak{h}}$ is an ideal of $\mathfrak{h}$; for let $r=x+k \in \mathfrak{r}$ with $x=r \pi_{\mathfrak{m}}, k=r \pi_{\mathfrak{h}}$, then for any $h \in \mathfrak{h},\left[\begin{array}{ll}k & h\end{array}\right]+\left[\begin{array}{ll}k & h\end{array}\right]=\left[\begin{array}{ll}r & h\end{array}\right] \in \mathfrak{r}$ which implies $\mathfrak{r}_{\mathfrak{m}}$ and $\mathfrak{x} \pi_{\mathfrak{h}}$ are $\mathfrak{h}$-invariant. But $\mathfrak{m}$ is $\mathfrak{h}$-irreducible therefore $\mathfrak{r}_{\mathfrak{m}}=0$ or $\mathfrak{m}$ and if $\mathfrak{r} \pi_{\mathfrak{m}}=0$, then $0 \neq \mathfrak{r}=\mathfrak{r} \pi_{\mathfrak{h}} \subset \mathfrak{h}$ which implies $\mathfrak{r}$ is a solvable ideal of $\mathfrak{h}$. Thus we must have $\mathfrak{r}_{\mathfrak{m}}=\mathfrak{m}$. Next let $x, y \in \mathfrak{m}=\mathfrak{r}_{\mathfrak{m}}$, then there exists $k \in \mathfrak{h}$ so that $x+k \in \mathfrak{r}$ and since $\mathfrak{r}$ is an ideal, $y x+[y k]+h(y, x)=[y x+k] \in \mathfrak{r}$. Thus for any $h(x, y) \in \mathfrak{h}=\mathfrak{h}(\mathfrak{m}, \mathfrak{m}), z=y x+[y k] \in \mathfrak{m}=\mathfrak{r} \pi \mathfrak{m}$ is such that $z+h(x, y) \in \mathfrak{r}$ and therefore $\mathfrak{h}=\mathfrak{h}(\mathfrak{m}, \mathfrak{m})=\mathfrak{x} \pi_{\mathfrak{h}}$.

Next let $p=x+h \in \mathfrak{g}$ and let $k \in \mathfrak{h}=\mathfrak{x}_{\mathfrak{h}}$ so that $x+k \in \mathfrak{r}$, then $p=(x+k)$ $+(h-k) \in \mathfrak{r}+\mathfrak{h}$ so that $\mathfrak{g}=\mathfrak{r}+\mathfrak{h}$. Also $\mathfrak{r} \cap \mathfrak{h}=0$ since $\mathfrak{h}$ is semi-simple. Next let $z \in \mathfrak{m}=\mathfrak{r} \pi \mathfrak{m}$ so that $z+h \in \mathfrak{r}$, then $p=(x-z)+(z+h) \in \mathfrak{m}+\mathfrak{r}$ i.e. $\mathfrak{g}=$ $\mathfrak{m}+\mathfrak{r}$ and also $\mathfrak{m} \cap \mathfrak{r}=0$ : otherwise $\mathfrak{m} \cap \mathfrak{r}$ is a nonzero $\mathfrak{h}$-invariant subspace of $\mathfrak{m}$ and therefore equals $\mathfrak{m}$; thus $\mathfrak{m}=\mathfrak{m} \cap \mathfrak{r} \subset \mathfrak{r}$ and $\mathfrak{g}=\mathfrak{m}+\mathfrak{r} \subset \mathfrak{r}$ which implies the semi-simple algebra $\mathfrak{h}$ is solvable.

Now in the above $\mathfrak{r}$ is an arbitrary nonzero solvable ideal so that from 
$\mathfrak{g}=\mathfrak{m} \dot{+} \mathfrak{r}=\mathfrak{m} \dot{+} \mathfrak{h}$ we have dim. $\mathfrak{r}=\operatorname{dim} . \mathfrak{h}$. Thus in particular for $\mathfrak{g}=$ radical of $\mathfrak{g}$ we have if $[\mathfrak{g} \mathfrak{g}] \neq 0$ that $\operatorname{dim} .[\mathfrak{g} \mathfrak{g}]=\operatorname{dim} . \mathfrak{h}=\operatorname{dim} . \mathfrak{g}$ which implies $\mathfrak{g}$ is not solvable; thus $\left[\begin{array}{ll}\mathfrak{g} & \mathfrak{g}\end{array}\right]=0$.

Example. Let $\mathfrak{h}=\{e, f, h\}$ be the 3 -dimensional split simple Lie algebra and let $\mathfrak{m}=\left\{x_{0}, x_{1}, \ldots, x_{s}\right\}$ be an $\mathfrak{h}$-irreducible module so that $\mathfrak{g}=\mathfrak{m}+\mathfrak{h}$ is g.L.t.s. with radical. Then we must have dim. $\mathfrak{m}=3$ and we have the following anti-commutative multiplication table for $g$.

\begin{tabular}{c|cccccc} 
& $x_{0}$ & $x_{1}$ & $x_{2}$ & $h$ & $e$ & $f$ \\
\hline$x_{0}$ & 0 & $-4 t x_{0}-2 t^{2} e$ & $-4 t x_{1}-2 t^{2} h$ & $2 x_{0}$ & 0 & $x_{1}$ \\
$x_{1}$ & & 0 & $-4 t x_{2}-4 t^{2} f$ & 0 & $-2 x_{0}$ & $x_{2}$ \\
$x_{2}$ & & 0 & $-2 x_{2}$ & $-2 x_{1}$ & 0 \\
$h$ & $0 \neq t \in F$ & & & 0 & $-2 e$ & $2 f$ \\
$e$ & & & & & 0 & $h$ \\
$f$ & & & & & & 0
\end{tabular}

We have $\left\{=\left\{c_{0}, c_{1}, c_{2}\right\}\right.$ where $c_{0}=x_{0}+t e, c_{1}=x_{1}+t h, c_{2}=x_{2}+2 t f$ and $\mathfrak{m}$ is a simple Lie algebra and $\Sigma=\Delta$.

The results in this section apply to a L.t.s.; for example, from the formula $[p s q s]-[p q] s=0$ for a L.t.s implies the space $g_{2}=g_{1} s$ of Theorem 2 is actually a simple ideal of $g$. Also Theorem 3 shows the Lie algebra $\mathrm{Q}=R\left(J_{0}\right)+D$ of $\left[3\right.$, p. 145] is simple (and of type $\left.E_{6}[3,4]\right)$. Another application is to Lie admissible algebras [5]: $A$ is a Lie admissible algebra with multiplication $x y$ if $A^{-}$(which is the vector space $A$ with multiplication $x * y=x y-y x)$ is a Lie algebra. Now if $A$ is Lie admissible and $D\left(A^{-}\right)$is the derivation algebra of the Lie algebra $A^{-}$, then $\mathfrak{g}=A^{-} \oplus D\left(A^{-}\right)$ is a Lie algebra with multiplication $\left[a_{1}+D_{1} a_{2}+D_{2}\right]=a_{1} * a_{2}+a_{1} D_{2}-a_{2} D_{1}+$ $R\left(a_{1} * a_{2}\right)+\left[D_{1}, D_{2}\right]$ where $R(a)$ denotes right multiplication in $A^{-}$. This can be generalized to a reductive Lie admissible algebra by just demanding $\mathrm{g}=A^{-} \oplus D\left(A^{-}\right)$be a Lie algebra. For example if $A$ is alternative, then $A^{-}$is Malcev and we take $\left[a_{1} a_{2}\right]=a_{1} * a_{2}+D\left(a_{1}, a_{2}\right)$ where $D\left(a_{1}, a_{2}\right)=\left[R\left(a_{1}\right), R\left(a_{2}\right)\right]$ $+R\left(a_{1} * a_{2}\right)$. In this last example if $A$ has an identity element, then $A^{-}$ has a center and cannot be simple or $D\left(A^{-}\right)$-irreducible. Since it would be desirable to utilize a simple or irreducible algebra we introduce the anticommutative algebra $A^{0}=A^{-} / C$ where $C=$ center $A^{-}$and form the Lie 
algebra $\mathfrak{g}^{0}=A^{0} \oplus D\left(A^{0}\right)$. For example, let $A$ be the split Cayley-Dickson algebra, then $A^{0}$ is the simple 7-dimensional Malcev algebra. Reductive Lie admissible algebras will be considered in a future paper.

Let $M=G / H$ be a reductive homogeneous space with canonical connection $\nabla$ of the first kind [7]. Thus $\nabla$ is the unique $G$-invariant connection on $M$ with zero torsion and such that the projection of 1-parameter subgroups by $\pi: G \rightarrow G / H: a \rightarrow a H$ are geodesics in $M=G / H . \quad \nabla$ is uniquely determined by its value at $p_{0}=H \in M$ and is given by $\nabla_{X^{*}}\left(Y^{*}\right)_{p_{0}}=$ $\frac{1}{2} X Y\left(=\frac{1}{2}[X Y]_{\mathfrak{m}}\right)$ where $X, Y \in \mathfrak{m}$ and $X^{*}, Y^{*}$ are certain vector fields defined on a suitable neighborhood $N^{*}$ of $p_{0}$; see [7, p. 42]. Now in case $G / H$ is a Lie group, the multiplication $X Y$ becomes multiplication in the Lie algebra $\mathfrak{m}$ and this is determined by the group multiplication in $G / H$ at $p_{0}$ i.e. determined by the tangent vector to the commutator curve at $p_{0}$. We shall now define a local group-like (nonassociative) multiplication so that the multiplication $X Y=2 \nabla_{X^{*}}\left(Y^{*}\right)_{p_{0}}$ is obtained from a commutator curve.

From $[2,7]$ we can find a symmetric compact normal neighborhood $\mathfrak{U}$ of 0 in $\mathfrak{m}$ so that $N^{*}=\pi \exp \mathfrak{U}$ is a normal neighborhood of $p_{0}$ in $M$ diffeomorphic to $\mathfrak{U}$. For certain elements $a, b \in N^{*}$ we define a multiplication $a \cdot b$ on $N^{*}$ which is analytic when defined and satisfies

(1) There exists $a$ two-sided identity element $e$ in $N^{*}$

(2) For every $a \in N^{*}$, there exists $a$ unique two-sided inverse $a^{-1}$ $\left(a \cdot a^{-1}=a^{-1} \cdot a=e\right)$ and $a \rightarrow a^{-1}$ is analytic.

Thus $N^{*}$ is a local analytic hoop [10] and furthermore $\mathfrak{m}$ is the tangent algebra of $N^{*}$; that is, $\mathfrak{m}$ is the tangent space at the identity with multiplication of two tangent vectors defined by the tangent to the commutator curve through the identity just as in Lie group theory. For example the simple Moufang loop (= hoop with cancellation laws) obtained from the Cayley-Dickson algebra has the simple seven dimensional Malcev algebra as its tangent algebra.

For the construction of this multiplication let $a=\pi \exp X, b=\pi \exp Y$ where $X, Y \in \mathfrak{U}$ and define

$$
a \cdot b=\pi \exp \left(X+Y+\frac{1}{2} X Y\right)
$$

where as usual $X Y=[X Y]_{\mathfrak{m}}$. Clearly $e=\pi \exp 0$ and $a^{-1}=\pi \exp (-X)$ is 
the unique inverse of $a$; also the multiplication and the map $a \rightarrow a^{-1}$ are analytic. To show $\mathfrak{m}$ is the tangent algebra of $N^{*}$ we shall show $X Y$ equals the tangent vector to $z(t)$ at $t=0$ where $z\left(t^{2}\right)=[\pi \exp t X \cdot \pi \exp t Y]$. $[\pi \exp t Y \cdot \pi \exp t X]^{-1}$ for $t$ near 0 . But a straightforward computation shows $z\left(t^{2}\right)=\pi \exp \left(t^{2} X Y+0\left(t^{3}\right)\right)$ which yields the results. It should also be noted that since $G / H$ is reductive there exists a neighborhood [7, p. 42] $N_{1}^{*} \subset N^{*}$ of $p_{0}$ so that for any $h \in H$, the map $\phi(h): N_{1}^{*} \rightarrow N^{*}: \pi \exp X \rightarrow \pi\left(h(\exp X) h^{-1}\right)$ is an automorphism of the local hoop $N^{*}$ up to a third order approximation i.e. $\Phi(h)(\pi \exp X) \cdot \phi(h)(\pi \exp Y)=\phi(h)(\pi \exp X \cdot \pi \exp Y) \cdot \pi \exp (\varepsilon) \quad$ where $\varepsilon$ involves 3rd order products of $X$ and $Y$.

Let $G / H$ be a simply connected reductive space with canonical connection of the first kind, then in [11] it is shown that if $G / H$ is not symmetric (i.e. $\mathfrak{m m} \neq 0$ ), then the Lie algebra, $L(\mathfrak{m})$, generated by all left multiplications $L(X): \mathfrak{m} \rightarrow \mathfrak{m}: Y \rightarrow X Y$ equals the Lie algebra of the holonomy group if and only if the derivations $D(X, Y) \in L(\mathfrak{m})$ for all $X, Y \in \mathfrak{m}$; that is, $D(X, Y)$ are inner [12]. Also in [11] there is a correspondence between simple algebras $\mathfrak{m}$ and holonomy irreducible spaces $G / H$ which are not symmetric. Thus for example if $G / H$ is a Riemannian non-symmetric reductive space, then $G / H$ is holonomy irreducible if and only if $\mathfrak{m}$ is a simple algebra; in this case the Lie algebra of the holonomy group equals $L(\mathfrak{m})$ which is semi-simple. Consequently the holonomy group of $G / H$ and the group-like multiplication on $G / H$ are related by the tangent algebra briefly as follows. For $X, Y \in \mathfrak{m}, \exp t L(X)$ is in the holonomy group for $t$ near 0 and for $z(t)$ the commutator curve of the preceding paragraph we have at $t=0$

$$
\frac{d}{d t}[(\exp t L(X))(Y)]=X Y=\frac{d}{d t} z(t)
$$

which is what is obtained in case $G / H$ is a Lie group i.e. $H$ normal.

It should also be noted that the assumption of the field $F$ to be algebraically closed can be realized by a $G$-invariant almost complex structure $J$ on $G / H$ with $\nabla J=0$.

2. Simplicity of $\mathrm{m}$. To prove the results concerning the simplicity of $\mathfrak{m}$ we need the following lemma concerning ideals and derivations.

Lemma 5. Let $A$ be a finite dimensional nonassociative algebra over a field of 
characteristic zero such that $A^{2} \neq 0$. If $A$ has a proper ideal, then $A$ has a proper $D(A)$-invariant ideal where $D(A)$ is the derivation algebra of $A$.

Proof. Assume $A$ has no proper $D(A)$-invariant ideals and let $\mathfrak{L}=\mathfrak{L}(A)$ be the Lie algebra generated by all left and right multiplications $x L(a)=a x$ and $x R(a)=x a$ for all $a \in A$. Since $A \mathbb{\Omega}$ is a nonzero $D(A)$-invariant ideal of $A$ (noting $[D, \mathbb{R}] \subset \mathfrak{L}$, see [12]), $A \mathbb{\Omega}=A$. Now let $\mathfrak{S}$ be the radical of $\mathfrak{L}$, then for any $D \in D(A)$ we have $\widetilde{D}: \mathfrak{\Omega} \rightarrow \mathfrak{\Omega}: X \rightarrow[X, D]$ is a derivation of $\mathfrak{L}$ and

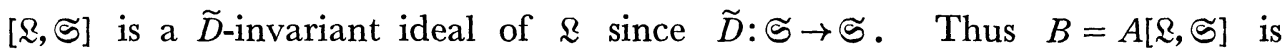
a $D(A)$-invariant ideal of $A$ (since $[[\Omega, \subseteq], R(x)] \subset[\Omega, \mathfrak{S}]$ and $[\Omega, \subseteq] \widetilde{D}=$ $[[\Omega, \subseteq], D] \subset[\Omega, \subseteq])$ and therefore $B=0$ or $B=A$. If $B=A$, then since $[\Omega, \subseteq] \subset \Re$ which is the radical of $\mathfrak{\Omega}^{*}$, where $\mathfrak{R}^{*}=$ associative enveloping algebra of $\mathbb{\Omega}[3, \mathrm{p}$. 45], we have $A=B=A[\Omega, \mathbb{S}] \subset A \Re \subset A$. This yields $A=A \Re$ which implies by induction that $A=0$; [3, p. 47]. Thus $B=0$ so that $[\Omega, \subseteq]=0$ and therefore $\mathfrak{S}$ is the center $\mathfrak{C}$ of $\mathfrak{L}$. We shall now show the linear transformations in $\mathbb{5}$ are semi-simple, then from Levi's theorem [3, p. 91] and [3, p. 81] we have $\&$ is completely reducible in $A$. Thus let $A \in \mathbb{C}$ and suppose $A$ is not semi-simple i.e. its minimum polynomial is not a product of distinct prime polynomials; this is equivalent to the fact that the enveloping algebra $\{A\}^{+}$has a nonzero nilpotent element $Z$. Then since $Z$ is a polynomial in $A \in \mathbb{C}$ we have $[R(x), Z]=[L(x), Z]=0$ so that for every element in $\mathfrak{L}$ and consequently for every $Q \in \mathfrak{L}^{*},[Q, Z]=0$. This implies that $Q Z$ and $Z Q$ are nilpotent so that $Z \in \Re=$ radical of $\mathfrak{L}^{*}$. But since $\Re$ is invariant under derivations of $\mathfrak{R}^{*}$ we have $\widetilde{D}: \Re \rightarrow \Re$ where $Q \widetilde{D}=$ $[Q, D]$ with $D \in D(A)$ i.e. $[\Re, D] \subset \Re$. This implies $A \Re$ is a $D(A)$-invariant ideal of $A$ which must be zero; thus $\Re=0$ so that $Z=0$ and the center $\subseteq$ consists of semi-simple linear transformations. Using $\mathbb{Q}$ is completely reducible and $A=A \Omega$ we write $A=\oplus \Sigma A_{j}$ where the $A_{j}^{\prime} s$ are $\mathbb{R}$-irreducible i.e. simple ideals of $A$. Since $A^{2} \neq 0$, there exists $A_{i}$ with $A_{i}^{2} \neq 0$; but $A_{i}^{2}$ is an \&-invariant subspace of $A_{i}$ so that $A_{i}=A_{i}^{2}$. This implies $A_{i}$ is a $D(A)$-invariant ideal for $A_{i} D=A_{i}^{2} D \subset A_{i} D \cdot A_{i}+A_{i} \cdot A_{i} D \subset A_{i}$ and therefore $A=A_{i}$ is simple i.e. $A$ has no proper ideals.

Next we use this lemma to investigate $\mathfrak{g}=\mathfrak{m}+\mathfrak{h}$ where $\mathfrak{m}$ is $\mathfrak{h}$-irreducible.

Theorem 6. Let $(\mathfrak{g}, \mathfrak{h}$ ) be a reductive pair with ( fixed) decomposition $\mathfrak{g}=\mathfrak{m}+\mathfrak{h}$ so that $\mathfrak{m}$ is $\mathfrak{h}$-irreducible and ad $\mathfrak{m} h=0$ implies $h=0$. If $\mathfrak{m m} \neq 0$, then $\mathfrak{m}$ is 
simple and the Lie transformation algebra $\mathfrak{L}=\mathfrak{\Omega}(\mathfrak{m})$ is semi-simple. Furthermore, if $F$ is the field of reals or is algebraically closed, then the derivation algebras $D(\mathfrak{m})$ and $a d \mathfrak{m} \mathfrak{h}$ are semi-simple and $D(\mathfrak{m}) \subset \mathfrak{R}$.

Proof. If $\mathfrak{m}$ has a proper ideal, then by Lemma 5 there exists a proper $\mathfrak{h}$-invariant subspace, a contradiction. Next from $[R(x), D]=R(x D)$ for any derivation $D$ of $\mathfrak{m}$, we see that $\{x \in \mathfrak{m}$ : trace $R(x)=0\}$ is a non-zero $\mathfrak{h}$-invariant subspace so that trace $R(x)=0$ for all $x \in \mathfrak{m}$. Thus from the fact that $\mathfrak{L}=\mathfrak{M}_{1}+\mathfrak{M}_{2}+\cdots$ where $\mathfrak{M R}_{1}=R(\mathfrak{m})$ and $\mathfrak{M}_{i}=\left[\mathfrak{M C}_{i-1}, \mathfrak{M}_{1}\right]$, see [12], we have trace $S=0$ for all $S \in \mathbb{R}$. To show $\mathfrak{R}$ is semi-simple we have that since $\mathfrak{m}$ is simple, $\mathfrak{L}=\mathfrak{C} \oplus \mathfrak{R}^{\prime}$ is completely reducible in $\mathfrak{m}$ where $\mathfrak{R}^{\prime}$ is semi-simple and $\mathfrak{E}$ is the center of $\mathfrak{R}$, see [3]. Now $\mathfrak{C} \subset \Gamma(\mathfrak{m})=\{S \in \operatorname{Hom}(\mathfrak{m}, \mathfrak{m}):[S, R(x)]$ $=0\}$ is the centroid of $\mathfrak{m}$, see [3] and since $\mathfrak{m}$ is simple $\Gamma(\mathfrak{m})$ is a field. From $[S, R(x)]=0$ for $S \in \Gamma(\mathfrak{m})$ we obtain $S R(x)=R(x S)$ so that $\Gamma(\mathfrak{m}) \mathbb{R} \subset \mathbb{R}$. Consequently, if $0 \neq A \in \mathfrak{S}$, then $I=A^{-1} A \in \Gamma(\mathfrak{m}) \mathbb{R}$ and we obtain the contradiction trace $I=0$.

Next assume $F$ is the field of reals since the case $F$ algebraically closed is similar to this case when the dim.m is odd. Since $\mathfrak{m}$ is $\mathfrak{h}$ irreducible, $H=a d \mathfrak{m} \mathfrak{h} \neq 0$ is completely reducible in $\mathfrak{m}$ and equals $H_{0} \oplus H^{\prime}$ where $H_{0}$ is the center of $H$ and $H^{\prime}$ semi-simple. If $D \in H_{0}$ and if dim. $m$ is odd, then $D$ has a characteristic root $\alpha$ and $\{x \in \mathfrak{m}: x(\alpha I-D)=0\}$ is a nonzero $\mathfrak{h}$-invariant subspace of $\mathfrak{m}$. This yields $D=\alpha I$ which is impossible for a non-zero derivation. Now if the $\operatorname{dim} . \mathfrak{m}$ is even, the characteristic polynomial has only quadralic factors (a linear factor yields the preceding case). Therefore if $q(\xi)=\xi^{2}+a \xi+b$ is such a factor we have that $\{x \in \mathfrak{m}: x q(D)=0\}$ is a non-zero $h$-invariant subspace so that $D^{2}+a D+b I=0$.

Using this formula we have for any $x, y \in \mathfrak{m}$ that

$$
\begin{aligned}
-b x y & =x y\left(D^{2}+a D\right) \\
& =2 x D \cdot y D-2 b x y
\end{aligned}
$$

and therefore $b x y=2 x D \cdot y D$. Now replacing $y$ by $y D$ in this last equation and using the quadratic equation for $D$ we obtain

$$
\begin{aligned}
b x \cdot y D & =-2 a x D \cdot y D-2 b x D \cdot y \\
& =-a b x y-2 b x D \cdot y .
\end{aligned}
$$

Interchanging $x$ and $y$ in this last equation and adding the results we obtain 


$$
\begin{aligned}
(x y) D & =x D \cdot y+x \cdot y D \\
& =-2 a x y+2(y D \cdot x+y \cdot x D) \\
& =-2 a x y+2(y x) D .
\end{aligned}
$$

Thus since $\mathfrak{m}^{2}=\mathfrak{m}$ we obtain $3 D=-2 a I$, a contradiction. Thus $H_{0}=0$ so that $a d_{\mathfrak{m}} \mathfrak{f}$ is semi-simple. A similar argument shows $D(\mathfrak{m})$ is semi-simple since any $D(\mathfrak{m})$-invariant subspace is also $\mathfrak{h}$-invariant.

The fact that $D(\mathfrak{m}) \subset \mathfrak{\Omega}$ will be proven by the same argument as that in the proof of Theorem 8 noting that since $\mathcal{L}$ and $D(\mathfrak{m})$ are semi-simple the traces of their elements are zero.

It is natural to consider the case when $\mathrm{g}$ is semi-simple, but Theorems 2 and 3 indicate that one should consider the general case of a reductive pair $(\mathfrak{g}, \mathfrak{h})$ with both $\mathfrak{g}$ and $\mathfrak{h}$ simple Lie algebras; see Theorem 8 .

Lemma 7. Let $(\mathfrak{g}, \mathfrak{h})$ be a reductive pair with $\mathfrak{g}$ and $\mathfrak{h}$ simple Lie algebras and a decomposition $\mathfrak{g}=\mathfrak{m}+\mathfrak{h}$ such that $\mathfrak{m m} \neq 0$. If $\mathfrak{m}$ has a proper ideal, then $\mathfrak{m}$ has a proper maximal ad $\mathfrak{m} \mathfrak{h}$-invariant ideal it which is a nilpotent Lie subalgebra and $\mathfrak{h}(\mathfrak{i t}, \mathfrak{n t})=\mathfrak{h}\left(\mathfrak{t}^{2}, \mathfrak{m}\right)=0$.

Proof. Since $\mathfrak{g}$ is simple, we have $\mathfrak{g}=\mathfrak{m}+\mathfrak{h}(\mathfrak{m}, \mathfrak{m})$. Now if $\mathfrak{m}$ has a proper ideal, it has a proper $D(\mathfrak{m})$-invariant ideal which is therefore $a d_{\mathfrak{m}} \mathfrak{g}$ invariant (= h-invariant); thus there exists a maximal $\mathfrak{h}$-invariant ideal $\mathfrak{t t}$. Next suppose $\mathfrak{h}(\mathfrak{n}, \mathfrak{n t}) \neq 0$, then $\mathfrak{t}+\mathfrak{h}(\mathfrak{n t}, \mathfrak{t})$ is a proper ideal of $\mathfrak{g}$. First $[\mathfrak{n}+\mathfrak{h}(\mathfrak{n}, \mathfrak{n}) \mathfrak{h}] \subset[\mathfrak{n} \mathfrak{h}]+[\mathfrak{h}(\mathfrak{n}, \mathfrak{n}) \mathfrak{h}] \subset \mathfrak{n}+\mathfrak{h}(\mathfrak{n}, \mathfrak{n})$, using (5). Next $[\mathfrak{n}+\mathfrak{h}(\mathfrak{n}, \mathfrak{n}) \mathfrak{m}] \subset \mathfrak{n} \mathfrak{m}$ $+\mathfrak{H}(\mathfrak{l t}, \mathfrak{n t})+[\mathfrak{h}(\mathfrak{l t}, \mathfrak{n}) \mathfrak{n t}] \subset \mathfrak{n}+\mathfrak{H}(\mathfrak{l t}, \mathfrak{n})$, using (3) and $\mathfrak{t t}$ is an $\mathfrak{h}$-invariant ideal. But from $(5)$ and $\mathfrak{h}(\mathfrak{t}, \mathfrak{n t}) \supset \mathfrak{h}(\mathfrak{t}, \mathfrak{t}) \neq 0$ we see that both of these are nonzero ideals of $\mathfrak{h}=\mathfrak{h}(\mathfrak{n t}, \mathfrak{m})$ and therefore $\mathfrak{h}=\mathfrak{h}(\mathfrak{t}, \mathfrak{t})=\mathfrak{h}(\mathfrak{m}, \mathfrak{t})$. Thus we finally obtain $[\mathfrak{t}+\mathfrak{h}(\mathfrak{t}, \mathfrak{t}) \mathfrak{m}] \subset \mathfrak{t}+\mathfrak{h}(\mathfrak{t}, \mathfrak{a t})$; this contradicts $g$ is simple and consequently we must have $\mathfrak{h}(\mathfrak{t}, \mathfrak{t})=0$. Using this we obtain from (3) and (4) that $J(\mathfrak{l t}, \mathfrak{n t}, \mathfrak{t})=0$ and $\mathfrak{h}\left(\mathfrak{t} \mathfrak{t}^{2}, \mathfrak{n t}\right)=0$.

Next $\mathfrak{h}(\mathfrak{t}, \mathfrak{n t}) \neq 0$, otherwise $\mathfrak{i t}$ is a proper ideal of $\mathfrak{g}=\mathfrak{m}+\mathfrak{h}:[\mathfrak{l t} \mathfrak{m}]=$ $\mathfrak{n t h}+\mathfrak{h}(\mathfrak{l t}, \mathfrak{n}) \subset \mathfrak{t}$ and $[\mathfrak{n} \mathfrak{h}] \subset \mathfrak{t}$. Now if $\mathfrak{n}=\mathfrak{t}^{2}$ we have from (4) that $\mathfrak{h}=\mathfrak{h}(\mathfrak{n}, \mathfrak{m})=\mathfrak{H}\left(\mathfrak{t}^{2}, \mathfrak{m}\right)=0$, a contradiction; thus $\mathfrak{t} \supset \mathfrak{t}^{2}$ (properly). Letting $\mathfrak{n t}^{1}=\mathfrak{n}, \mathfrak{t}^{2}=\mathfrak{n t h}, \mathfrak{n}^{k}=\mathfrak{n}^{k-1} \mathfrak{n}$ we see that $\mathfrak{n t}^{k}$ is an $\mathfrak{h}$-invariant subalgebra of $\mathfrak{m}$. Now since $\mathfrak{h}$ is simple, $\mathfrak{m}$ is $\mathfrak{h}$-completely reducible so that there exists an $\mathfrak{h}$-invariant subspace $\mathfrak{H}^{\prime}$ such that $\mathfrak{m}=\mathfrak{t}+\mathfrak{t}^{\prime}$. For this fixed decomposition we have 


$$
\mathfrak{n}^{k} \mathfrak{n}^{\prime} \subset \mathfrak{n}^{k-1}
$$

For $\mathfrak{n}^{2} \mathfrak{n}^{\prime} \subset \mathfrak{H}^{\prime} \subset \mathfrak{t}=\mathfrak{n}^{1}$ and assume for $k>2$ that $\mathfrak{n}^{k} \mathfrak{t}^{\prime} \subset \mathfrak{n}^{k-1}$, then using $\mathfrak{h}\left(\mathfrak{t}^{2}, \mathfrak{m}\right)=0$ and (3) we obtain

$$
J\left(\mathfrak{H}^{k}, \mathfrak{l t}, \mathfrak{H}^{\prime}\right)=\left[\mathfrak{H}^{k} \mathfrak{h}\left(\mathfrak{l t}, \mathfrak{H}^{\prime}\right)\right] \subset \mathfrak{H t}^{k} .
$$

This yields $\left(\mathfrak{t}^{k} \mathfrak{t}\right) \mathfrak{t}^{\prime} \subset \mathfrak{t}^{k}+\left(\mathfrak{n} \mathfrak{t}^{\prime}\right) \mathfrak{t}^{k}+\left(\mathfrak{t}^{\prime} \mathfrak{t}^{k}\right) \mathfrak{t}$ and using the induction hypothesis we have $\left(\mathfrak{t}^{k} \mathfrak{t}\right) \mathfrak{l t}^{\prime} \subset \mathfrak{t}^{k}$ i.e. $\mathfrak{t}^{k+1} \mathfrak{t}^{\prime} \subset \mathfrak{t}^{k}$. Next suppose that in the descending chain $\mathfrak{t} \supset \mathfrak{n}^{2} \supset \mathfrak{n}^{3} \supset \cdots$ there exists $q>2$ such that $0 \neq \mathfrak{n}^{q-1}=\mathfrak{n}^{q}\left(=\mathfrak{n}^{q-1} \mathfrak{n}\right)$, then $\mathfrak{t}^{q-1}$ is a proper ideal of $\mathfrak{g}$. For using $\mathfrak{g}=\mathfrak{n}+\mathfrak{t}^{\prime}+\mathfrak{h}$ we have $\left[\mathfrak{n}^{q-1} \mathfrak{h}\right] \subset \mathfrak{n}^{q-1} ;\left[\mathfrak{n t}^{q-1} \mathfrak{n}\right] \subset \mathfrak{n}^{q-1} \mathfrak{n}+\mathfrak{h}\left(\mathfrak{i t}^{q-1}, \mathfrak{n}\right) \supset \mathfrak{n}^{q} ;$ and $\left[\mathfrak{n}^{q-1} \mathfrak{n}^{\prime}\right] \subset \mathfrak{n}^{q-1} \mathfrak{n}^{\prime}=\mathfrak{n}^{q} \mathfrak{n}^{\prime} \subset \mathfrak{n}^{q-1}$, using $\mathfrak{t t}^{q-1}=\mathfrak{t t}^{q}$ and $\mathfrak{t}^{k} \mathfrak{t t}^{\prime} \subset \mathfrak{t}^{k-1}$. This contradiction shows we must have $\mathfrak{n}^{k-1} \subset \mathfrak{n}^{k}$ (properly) which implies $\mathfrak{n}$ is nilpotent.

The next few pages are devoted to showing the ideal $\mathfrak{n}$ of Lemma 7 is such that $\mathfrak{n}^{2}=0$ and using the non-degenerate Killing form, $B(X, Y)$, of $\mathfrak{g}$ with dual space arguments to actually show that $\mathfrak{t}=0$; thus by Lemma 7 $\mathfrak{m}$ must be simple. First we observe $\mathfrak{n}=0$ if and only if its center $\mathfrak{c}$ is zero. So we concentrate on the $\mathfrak{h}$-invariant subalgebra $\mathfrak{c}$. Using $\mathfrak{h}$ is completely reducible in $\mathfrak{m}$ and $\mathfrak{i t}$ (since $\mathfrak{h}$ is simple) we write $\mathfrak{t}=\mathfrak{c}+\mathfrak{c}_{1}$ where $\mathfrak{c}_{1}$ is $\mathfrak{h}$-invariant and $\mathfrak{m}=\mathfrak{t}+\mathfrak{t}^{\prime}=\mathfrak{c}+\mathfrak{c}_{1}+\mathfrak{t}^{\prime}$ so that $\mathfrak{g}=\mathfrak{c}+\mathfrak{c}_{1}+\mathfrak{t}^{\prime}+\mathfrak{h}$. We now compute $a d_{g} X$ for $X$ in these various spaces:

$$
\begin{aligned}
& a d_{9} x=\left(\begin{array}{cccc}
0 & 0 & 0 & 0 \\
0 & 0 & 0 & 0 \\
X_{31} & X_{32} & 0 & X_{34} \\
X_{41} & 0 & 0 & 0
\end{array}\right) \quad \text { for } x \in \mathfrak{c}, \\
& a d_{9} y=\left(\begin{array}{cccc}
0 & 0 & 0 & 0 \\
Y_{21} & Y_{22} & 0 & 0 \\
Y_{31} & Y_{32} & 0 & Y_{34} \\
0 & Y_{42} & 0 & 0
\end{array}\right) \text { for } y \in \mathfrak{c}_{1}, \\
& a d_{9} z=\left(\begin{array}{cccc}
Z_{11} & Z_{12} & 0 & Z_{14} \\
Z_{21} & Z_{22} & 0 & Z_{24} \\
Z_{31} & Z_{32} & Z_{33} & Z_{34} \\
0 & 0 & Z_{43} & 0
\end{array}\right) \text { for } z \in \mathfrak{t}^{\prime},
\end{aligned}
$$




$$
a d_{\mathrm{g}} h=\left(\begin{array}{cccc}
H_{11} & 0 & 0 & 0 \\
0 & H_{22} & 0 & 0 \\
0 & 0 & H_{33} & 0 \\
0 & 0 & 0 & H_{44}
\end{array}\right) \quad \text { for } h \in \mathfrak{h} ;
$$

where these block matrices are computed from the various facts $\mathfrak{c}^{2}=0$, $\mathfrak{n}=\mathfrak{c}+\mathfrak{c}_{1}$ is an ideal of $\mathfrak{m}$, the spaces are $\mathfrak{h}$-invariant and $\mathfrak{h}(\mathfrak{h}, \mathfrak{n})=0$. From these matrices we obtain $B(x, \mathfrak{c})=B\left(x, \mathfrak{c}_{1}\right)=B(x, \mathfrak{h})=0$ and $B(x, z)=$ trace $X_{34} Z_{43}+$ trace $X_{41} Z_{14}$. We compute these traces as follows. For any $x, y \in \mathfrak{m}$ and $h \in \mathfrak{h}$ we define linear transformations $\varepsilon(x)$ and $\delta(x)$ by

$$
y \varepsilon(x)=h(y, x)
$$

and

$$
h \delta(x)=\left[\begin{array}{ll}
x & h
\end{array}\right]=-h a d_{g} x
$$

where $\varepsilon(x): \mathfrak{m} \rightarrow \mathfrak{h}$ and $\delta(x): \mathfrak{h} \rightarrow \mathfrak{m}$. From this we see the matrix $X_{34}$ corresponds to $\varepsilon(x)$ acting in $\mathfrak{n}^{\prime}, Z_{14}$ corresponds to $\varepsilon(z)$ acting in $\mathfrak{c},-X_{41}$ and $-Z_{43}$ correspond to $\delta(x)$ and $\delta(z)$ acting in $\mathfrak{h}$. Thus to compute the matrix $X_{34} Z_{43}$ and $Z_{14} X_{41}$ we let $s \in \mathfrak{n}^{\prime}$ and $t \in \mathfrak{c}$, then

$$
s \varepsilon(x) \delta(z)=[z h(s, x)] \equiv s \sigma(x, z)
$$

yields the matrix $-X_{34} Z_{43}$ and

$$
t \varepsilon(z) \delta(x)=[x h(t, z)] \equiv t \sigma(z, x)
$$

yields the matrix $-Z_{14} X_{41}$.

Now we shall show $\mathfrak{t}^{2}=0$. For $x \in \mathfrak{c} \cap \mathfrak{t}^{2}, s \in \mathfrak{t}^{\prime}$ and $z \in \mathfrak{t}_{1}$ we have

$$
s \varepsilon(x) \delta(z)=[z h(s, x)] \in\left[\mathfrak{n}^{\prime} \mathfrak{h}\left(\mathfrak{n}^{2}, \mathfrak{m}\right)\right]=0,
$$

thus $X_{34} Z_{43}$ is zero. Next from (3) we have for $t \in \mathfrak{c}$ that

$$
\begin{aligned}
& J(x, t, z)=\left[\begin{array}{ll}
x & h(t, z)]
\end{array}\right]\left[\begin{array}{ll}
t & h(z, x)
\end{array}\right]+[z h(x, t)]
\end{aligned}
$$

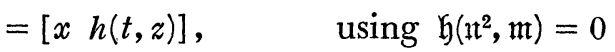

$$
\begin{aligned}
& =t \varepsilon(z) \delta(x) \text {. }
\end{aligned}
$$

But since $t, x \in \mathfrak{c} \subset \mathfrak{n}$ (an ideal) we see $J(x, t, z)=0$ so that $Z_{14} X_{41}$ is zero. This proves $B(x, \mathfrak{g})=0$ so that $\mathfrak{c} \cap \mathfrak{t}^{2}=0$. Now let $\mathfrak{n} \supset \mathfrak{t}^{2} \supset \cdots \supset \mathfrak{n}^{k} \supset$ $\mathfrak{n}^{k+1}=0$, then $\mathfrak{n}^{k} \subset \mathfrak{c} \cap \mathfrak{n}^{2}$ if $k \geqq 2$ so that $\mathfrak{n}^{k}=0$ if $k \geqq 2$ i.e. $\mathfrak{n}^{2}=0$. Thus $\mathfrak{t}=\mathfrak{c}$ and therefore we have $\mathfrak{m}=\mathfrak{c}+\mathfrak{t}^{\prime}$ with $B(\mathfrak{c}, \mathfrak{c})=B(\mathfrak{c}, \mathfrak{h})=0$ and $\mathfrak{h}=\mathfrak{h}(\mathfrak{c}, \mathfrak{m})$. 
Next from $\mathfrak{g}=\mathfrak{m}+\mathfrak{h}$ we have for any $z \in \mathfrak{m}$ that

$$
a d_{\mathfrak{g}} z=\left(\begin{array}{rc}
R(z) & \varepsilon(z) \\
-\delta(z) & 0
\end{array}\right)
$$

and since $g$ is simple, $0=\operatorname{tr} a d z=\operatorname{tr} R(z)$. Also since $\mathfrak{h}=[\mathfrak{G} h]$ is simple and $\mathfrak{h} \rightarrow a d_{\mathrm{c}} \mathfrak{h}: h \rightarrow a d_{\mathrm{c}} h$ and $h \rightarrow a d_{\mathfrak{m}} \mathfrak{h}: h \rightarrow a d_{\mathfrak{m}} h$ are representations we have $\operatorname{tr} a d_{\mathrm{c}} h=\operatorname{tr} a d_{\mathfrak{m}} h=0$ for all $h \in \mathfrak{h}$. From $\sigma(x, y)=\varepsilon(x) \delta(y)$ i.e. $z \sigma(x, y)=$ $[y h(z, x)]$ and from (3) we have

$$
[R(x), R(y)]-R(x y)=\sigma(x, y)-\sigma(y, x)+a d_{\mathfrak{m}} h(x, y) .
$$

Thus if $r(x, y)=\operatorname{tr} \sigma(x, y)$ we have $r(x, y)=r(y, x)$ and from this we obtain from the matrix for $a d_{g} z$ that

$$
\begin{aligned}
B(x, y) & =\operatorname{tr} a d_{\mathrm{g}} x a d_{\mathrm{g}} y \\
& =\operatorname{tr} R(x) R(y)-\operatorname{tr} \varepsilon(x) \delta(y)-\operatorname{tr} \delta(x) \varepsilon(y) \\
& =\operatorname{tr} R(x) R(y)-r(x, y)-r(y, x) \\
& =\operatorname{tr} R(x) R(y)-2 r(x, y) .
\end{aligned}
$$

Using this formula we shall show $B\left(\mathfrak{c}, \mathfrak{t t}^{\prime}\right)=0$ and from $B(\mathfrak{c}, \mathfrak{c})=B(\mathfrak{c}, \mathfrak{h})=0$ and $\mathfrak{g}=\mathfrak{c}+\mathfrak{n}^{\prime}+\mathfrak{h}$ we have $B(\mathfrak{c}, \mathfrak{g})=0$; thus $\mathfrak{c}=0$. Now for $x \in \mathfrak{m}, y \in \mathfrak{c}$ we see from the decomposition $\mathfrak{n}=\mathfrak{c}+\mathfrak{t}^{\prime}$ that the matrix for $R(x) R(y)$ has zeros on its diagonal so that $\operatorname{tr} R(x) R(y)=0$ and therefore

$$
B(x, y)=-2 r(x, y) \quad \text { for } x \in \mathfrak{m}, y \in \mathfrak{c} \text {. }
$$

We shall now show that for any $x \in \mathfrak{t t}^{\prime}$ that $r(x, y)=0$ for $y \in \mathfrak{c}$. From the decomposition $\mathfrak{m}=\mathfrak{c}+\mathfrak{t t}^{\prime}$ we have for $\sigma(x, y)=\varepsilon(x) \delta(y)$ with $x \in \mathfrak{t}^{\prime}, y \in \mathfrak{c}$ that

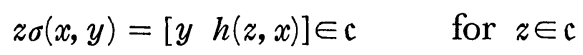

and

$$
z_{1} \sigma(x, y)=\left[\begin{array}{ll}
y & \left.h\left(z_{1}, x\right)\right] \in \mathfrak{c} \quad \text { for } z_{1} \in \mathfrak{n}^{\prime}
\end{array}\right.
$$

therefore $\sigma(x, y)$ has a matrix $A$ of the form

$$
A=\left(\begin{array}{ll}
A_{11} & 0 \\
A_{21} & 0
\end{array}\right)
$$

Thus $r(x, y)=\operatorname{tr} \sigma(x, y)=\operatorname{tr} A_{11}=\operatorname{tr} \sigma(x, y)$. Again with $x \in \mathfrak{n}^{\prime}$ and $y, z \in \mathfrak{c}$ we have from $(3), \mathfrak{h}(\mathfrak{c}, \mathfrak{c})=0, \mathfrak{c}^{2}=0$ and $\mathfrak{c}=\mathfrak{t}$ is an ideal of $\mathfrak{m}$, 


$$
\begin{aligned}
& 0=J(z, x, y) \\
& =[z h(x, y)]+[y \quad h(z, x)] \\
& =z[\operatorname{adc} h(x, y)+\sigma(x, y)] \text {. }
\end{aligned}
$$

Thus on $c$ we have

$$
0=\operatorname{adch}(x, y)+\sigma(x, y)
$$

and taking traces on $\mathfrak{c}$ we obtain $0=\operatorname{tr} c \sigma(x, y)=r(x, y)$ for all $x \in \mathfrak{t}^{\prime}, y \in \mathfrak{c}$ and therefore $B\left(\mathfrak{c}, \mathfrak{t}^{\prime}\right)=0$. This proves part of the following theorem.

Theorem 8. Let $(\mathfrak{g}, \mathfrak{h})$ be a reductive pair where $\mathfrak{g}$ and $\mathfrak{h}$ are simple Lie algebras with a decomposition $\mathfrak{g}=\mathfrak{m} \dot{+} \mathfrak{h}$ so that $\mathfrak{m} \mathfrak{m} \neq 0$. Then $\mathfrak{m}$ is a simple algebra and the Lie transformation algebra $\mathfrak{Q}$ is semi-simple and $a d_{\mathrm{mb}} \subset \mathbb{Q}$ i.e. consists of inner derivations.

The proof that $\mathfrak{Z}$ is semi-simple is similar to that in Theorem 6 and is omitted. Now let $D \in a d \mathfrak{m} \mathfrak{y} \equiv H$, then since $[R(x), D]=R(x D)$ we have $[\Omega, D] \subset \mathbb{R}$ so that the map $\tilde{D}: \Omega \rightarrow \Omega: X \rightarrow[X, D]$ is a derivation of $\mathbb{\Omega}$. But

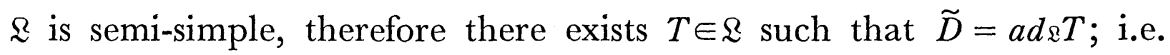

$$
[X, D]=X \widetilde{D}=\operatorname{Xad} \Omega=[X, T]
$$

for all $X \in \mathfrak{\Omega}$. Thus $[D-T, X]=0$ so that $D-T \in \Gamma(\mathfrak{m})=$ centroid of $\mathfrak{m}$ and $\Gamma(\mathfrak{n t})$ is a field, [3].

Let $D-T=U \in \Gamma(\mathfrak{m})$, then if $U \neq 0$ we have $I=D U^{-1}-T U^{-1}$ and $T U^{-1} \in \Gamma(\mathfrak{m}) L \subset L$ so that trace $T U^{-1}=0$. Thus to obtain a contradiction to $U \neq 0$ we shall show trace $D V=0$ for any $V \in \Gamma(\mathfrak{m})$. First we note for any $D \in H$ that $[D, V]=0$ for all $V \in \Gamma(\mathfrak{m})$. For from the Jacobi identity we have

$$
\begin{aligned}
{[[D, V], R(x)] } & =[[D, R(x)], V]+[D,[V, R(x)]] \\
& =-[R(x D), V]=0
\end{aligned}
$$

so that $[D, V]=W \in \Gamma(\mathfrak{m})$. If $W \neq 0$, then $I=(D V-V D) W^{-1}=D W^{-1} V-$ $V D W^{-1}$, using $\Gamma(\mathfrak{m})$ is commutative; from this equation trace $I=0$. Thus $[D, V]=0$. Now since $H=[H, H]$ is semi-simple we have that the given derivation $D=\Sigma\left[D_{i}, D_{j}\right]$ and therefore $D V=\sum\left[D_{i}, D_{j} V\right]$ so that trace $D V=0$.

Many useful examples can be computed if we take $\mathfrak{h}$ to be a regular simple subalgebra [1, p. 147] of $\mathfrak{g}$. Thus by definition there exists a Cartan subalgebra $\mathfrak{R}$ of $g$ such that if $g=\mathfrak{R}+\sum g_{\alpha}+\sum g_{-\alpha}$, where $\alpha \in \Delta=$ 
system of positive roots, is the Cartan decomposition of $g$ relative to $\Re$, then there exist a subsystem $\widetilde{\Delta} \subset \Delta$ and a corresponding subalgebra $\widetilde{\Re} \subset \Re$ such that $\mathfrak{h}=\widetilde{\Re}+\sum \mathfrak{g}_{\beta}+\sum \mathfrak{g}_{-\beta}, \beta \in \widetilde{\Delta}$. It is easy to show that $\mathscr{\Re}=\widetilde{\Re}+\mathfrak{R}^{\prime}$ where $\mathfrak{K}^{\prime}$ is such that $\left[\mathfrak{h} \mathfrak{\Re}^{\prime}\right]=0$ and from this if we let $\mathfrak{m}=\mathfrak{R}^{\prime}+\sum \mathfrak{g}_{\alpha} \dot{+} \mathfrak{g}_{-\alpha}$ with $\sigma \in \Delta-\tilde{\Delta}$, then $\mathfrak{g}=\mathfrak{m}+\mathfrak{h}$ is the desired decomposition of $\mathfrak{g}$. Using this decomposition we have the following explicit examples.

Example 1. Using the notation of [3] let $g$ be of type $A_{l}$, the $l+1 \times l+1$ matrices of trace zero, then $g$ has basis

$$
e_{i j} \quad(\text { for } i \neq j=1, \ldots, l+1)
$$

which form a basis for the nonzero root spaces and

$$
h_{k}=e_{k k}-e_{l+1, t+1} \quad k=1, \ldots, l
$$

which forms a basis for the Cartan subalgebra $\mathfrak{R}$. We shall let $\mathfrak{h}$ be of type $A_{l-1}$, since in this case $\mathfrak{m}$, in the decomposition $\mathfrak{g}=\mathfrak{m} \dot{+} \mathfrak{h}$, will have smallest dimension and the possibility of $a d \mathfrak{m} \mathfrak{h}$ equaling $D(\mathfrak{m})$ is best. As in the above remarks we let $\mathfrak{h}$ have basis $\left\{e_{i j}: i \neq j=2, \ldots, l+1\right.$ and $\left.h_{k} k=2, \ldots, l\right\}$ and let $\mathfrak{m}$ have basis $\left\{e_{1 j}, e_{i 1}, u: i, j=2, \ldots, l+1\right\}$ where $u=\sum_{i=1}^{l} x_{i} h_{i}$ is determined by choosing $x_{1}=-l, x_{2}=\cdots=x_{l}=1$. Then $\mathfrak{g}=\mathfrak{m} \dot{+} \mathfrak{h}$ is reductive and $\mathfrak{m}$ is simple. The anti-commutative multiplicative relations in $\mathfrak{m}$ for the above basis elements are

$$
\begin{gathered}
u e_{1 k}=-(l-1) e_{1 k}, u e_{k 1}=(l-1) e_{k 1}, \quad k=2, \ldots, l+1, \\
e_{1 p} e_{1 q}=0=e_{p 1} e_{q 1}, \\
e_{1 p} e_{q 1}=\frac{1}{l} \delta_{p q} u, \quad p, q=2, \ldots, l+1 .
\end{gathered}
$$

These are easily checked by computing the matrix commutators $[e f]=e f-f e \equiv e f+h(e, f)$.

Next to compute $D \in D(\mathfrak{m})$ let

$$
\begin{aligned}
u D & =a_{1} u+\sum a_{1 p} e_{1 p}+\sum b_{p 1}^{\prime} e_{p 1} \\
e_{1 i} D & =b_{i} u+\sum b_{i p} e_{1 p}+\sum b_{p_{i}}^{\prime} e_{p 1} \\
e_{j 1} D & =c_{j} u+\sum c_{j p} e_{1 p}+\sum c_{p j}^{\prime} e_{p 1}
\end{aligned}
$$

where $i, j, p=2, \ldots, l+1$. Then applying $D$ to the above multiplicative relations we find that $D$ has a matrix of the form 


$$
\left(\begin{array}{ccc}
0 & 0 & 0 \\
0 & b & 0 \\
0 & 0 & -b^{\prime}
\end{array}\right)
$$

where $b$ is the $l \times l$ matrix $\left(b_{i p}\right)$. Since the derivation $D^{\prime}$ with $b=I$ is a basis for the center of $D(\mathfrak{m})$ i.e. the Lie algebra of matrices as just computed, we can identify $D(\mathfrak{m})$ with $D^{\prime} F \oplus \mathfrak{h}$, noting that $\mathfrak{h}$ is the $l^{2}-1$ matrices of trace zero. Note that $D(\mathfrak{m})$ is not semi-simple even though $\mathfrak{m}$ is simple.

ExAmple 2. We consider the Lie algebra $\mathfrak{s} 0(n)$ of $n \times n$ skew-symmetric matrices and show directly that $\mathfrak{g}_{\mathfrak{D}}(n)=\mathfrak{m} \dot{+} \mathfrak{g}(n-2)$ is g.L.t.s. with $\mathfrak{h}=\mathfrak{g}_{\mathfrak{D}}(n-2)$ and $\mathfrak{m}$ simple. First for $p<n$ write $\mathfrak{g}_{\mathfrak{D}}(n)=\mathfrak{m} \dot{+} \mathfrak{g} \mathfrak{D}(p)$ as a Lie algebra as follows: identify $\mathfrak{g}_{0}(p)$ as the lower right-hand block of $p \times p$ skew-symmetric matrices in $\mathfrak{S}_{\mathfrak{D}}(n)$ and identify $\mathfrak{m}$ with the corresponding complementary set of matrices. Then $\mathfrak{s} \mathfrak{D}(n)=\mathfrak{m}+\mathfrak{s} \mathfrak{D}(p)$ is a g.L.t.s. and for $p=n-1, \mathfrak{m}^{2}=0 ;$ for $p=n-2, \mathfrak{m}^{2}=\mathfrak{m}$ and $\mathfrak{m}$ is simple. To see this let $e_{i j} i, j=1, \ldots, n$ denote the usual matrix basis, then from the above description of $\mathfrak{m}$ we see that $\mathfrak{m}$ has basis

$$
u=e_{12}-e_{21}, f_{j}=e_{1 j}-e_{j 1}, f_{j}^{\prime}=e_{2 j}-e_{j 2}
$$

for $j=3, \ldots, n$. The multiplicative relations for $\mathfrak{m}$ are

$$
\begin{array}{lr}
u f_{j}=-f_{j}^{\prime}, u f_{j}^{\prime}=f_{j} & j=3, \ldots, n \\
f_{j} f_{k}=0=f_{j}^{\prime} f_{k}^{\prime} & j, k=3, \ldots, n \\
f_{j} f_{k}^{\prime}=-\delta_{j k} u & j, k=3, \ldots, n .
\end{array}
$$

The proof in that $\mathfrak{m}$ is simple is briefly as follows. Suppose

$$
b=b_{0} u+\sum b_{j} f_{j}+\sum b_{j}^{\prime} f_{i}^{\prime} \in \mathfrak{b}
$$

where $\mathfrak{b}$ is an ideal of $\mathfrak{m}$ and $b_{p}, b_{q}^{\prime} \in F$. Then $b+u(u b)=b_{0} u \in \mathfrak{b}$ and if $b_{0} \neq 0$, then using the multiplicative relations $\mathfrak{b}=\mathfrak{m}$. If $b_{0}=0$, but some $b_{k} \neq 0$, say, then from the multiplicative relations we have

$$
\begin{aligned}
\tilde{b}=b f_{k}^{\prime} & =\sum_{j} b_{j} f_{j} f_{k}^{\prime}+\sum_{j} b_{j}^{\prime} f_{j}^{\prime} f_{k}^{\prime} \\
& =-b_{k} u=\widetilde{b}_{0} u \in \mathfrak{b}
\end{aligned}
$$

so that again $\mathfrak{m}=\mathfrak{b}$. Next to compute $D \in D(\mathfrak{m})$ let 


$$
\begin{aligned}
u D & =a u+\sum a_{p} f_{p}+\sum a_{p}^{\prime} f_{p}^{\prime} \\
f_{\imath} D & =b_{i} u+\sum b_{i p} f_{p}+\sum b_{i p}^{\prime} f_{p}^{\prime} \\
f_{j}^{\prime} D & =c_{j} u+\sum c_{j p} f_{p}+\sum c_{j p}^{\prime} f_{p}^{\prime}
\end{aligned}
$$

where $i, j, p=3, \ldots, n$. Then applying $D$ to the multiplicative relations for $\mathfrak{m}$ we see that $D$ has a matrix of the form

$$
\left(\begin{array}{ccc}
0 & 0 \cdots 0 & 0 \cdots 0 \\
0 & & \\
\vdots & \left(b_{\imath j}\right) & \left(b_{i j}^{\prime}\right) \\
0 & & \\
0 & & \\
\vdots & -\left(b_{i j}^{\prime}\right) & \left(b_{i j}\right) \\
0 & &
\end{array}\right)
$$

where $b_{i j}=-b_{j i}$ and $b_{i j}^{\prime}=b_{j i}^{\prime}$ are the coefficients in the expansion for $f_{i} D$ above. In this case $\mathfrak{h}$ is identified with diagonal block subalgebra and note that in both examples $D(\mathfrak{m})$ annihilates the subspace $u F$ so in particular is not irreducible on $\mathfrak{m}$; this is generally true by Theorem 7.5 of $[1, \mathrm{p} .161]$. These examples motivate the following corollary.

COROLlary 9. Let $(\mathfrak{g}, \mathfrak{h})$ be a reductive pair where $\mathfrak{h}$ is a maximal subalgebra of $\mathrm{g}$ and in the decomposition $\mathrm{g}=\mathfrak{m}+\mathfrak{h}, \mathfrak{m} \mathfrak{m} \neq 0$. Then $\mathfrak{m}$ is a simple algebra.

Proof. Suppose $\mathfrak{b}$ is a proper ideal of $\mathfrak{m}$, then from Lemma 5 we may assume $\mathfrak{b}$ is $a d_{\mathfrak{m}} \mathfrak{f}$-invariant. Next a straight-forward calculation shows that $\mathfrak{f}=\mathfrak{b}+\mathfrak{h}$ is a proper Lie subalgebra of $\mathfrak{g}$ containing $\mathfrak{h}$.

\section{BIBLIOGRAPHY}

[1] E.B. Dynkin, Semisimple subalgebras of semisimple Lie algebras, p. 111, Maximal subgroups of the classical groups, p. 245, Amer. Math. Soc. Translations, Ser. 2, 6 (1957).

[2] S. Helgason, Differential Geometry and Symmetric Spaces, Academic Press, New York, 1962.

[ 3 ] N. Jacobson, Lie Algebras, Wiley (Interscience), New York, 1962.

[4] - Exceptional Lie Algebras, mimeographed notes.

[ 5 ] P.J. Laufer and M.L. Tomber, Some Lie admissible algebras, Canad. J. Math., 14 (1962), p. 287. 
[6] W.G. Lister, A structure theory of Lie triple systems, Trans. Amer. Math. Soc., 72 (1952), p. 217.

[7] K. Nomizu, Invariant affine connections on homogeneous spaces, Amer. J. Math. 76 (1954), p. 33.

[8] A. Sagle, On simple extended Lie algebras over fields of characteristic zero, Pacific J. Math., 15 (1965), p. 621.

[9] - On anti-commutative algebras and general Lie triple systems; On simple algebras obtained from homogeneous general Lie triple systems, Pacific J. Math., 15 (1965), p. 281; p. 1397.

[10] - On anti-commutative algebras and analytic loops, Canadian J. Math., 17 (1965), p. 550.

[11] —, On anti-commutative algebras and homogeneous spaces, to appear. J. Math. Mech. (1967).

[12] R.D. Schafer, Inner derivations of non-associative algebras, Bull. Amer. Math. Soc., 55 (1949), p. 769.

[13] K. Yamaguti, On the Lie triple system and its generalization, J. Sci. of Hiroshima Univ., 21 (1958), p. 155.

[14] - On the theory of Malcev algebras, Kumamoto J. Sci., 6 (1963), p. 9.

University of Minnesota 